\title{
Hernia hiatal: un desafio diagnóstico y terapéutico. A propósito de un caso
}

\author{
José Julián Pérez Vaca* \\ Jairo Fabrizzio Viscarra Leon* \\ Cristina Gabriela Ríos Orozco**
}

\begin{abstract}
*Médico. Postgradista de Cirugía General. Residente de tercer año. Facultad de Postgrado. Escuela de Medicina. Universidad de Especialidades Espíritu Santo. Guayaquil. Ecuador.

**Médico. Postgradista de Medicina Interna. Residente de primer año. Facultad de Postgrado. Escuela de Medicina. Universidad de Especialidades Espíritu Santo. Guayaquil. Ecuador.

Correspondencia: Dr. José Julián Pérez, OMNI HOSPITAL Guayaquil - Ecuador. Dirección: Av. Juan Tanca Marengo y 13E. Tel: 0996164901. Correo electrónico: jose.julian.perez.vaca@gmail.com
\end{abstract}

\section{RESUMEN}

La presentación clínica de una hernia hiatal, constituye un verdadero desafío diagnóstico y terapéutico para el personal de salud, debido a que la presencia de reflujo gastroesofágico no es sinónimo de hernia hiatal y por otro lado la hernia hiatal no siempre debuta con reflujo gastroesofágico, sino más bien se manifiesta con sintomatología extra-abdominal como dolor torácico o manifestaciones pulmonares. A continuación se presenta un caso clínico de una paciente de 66 años de edad con diagnóstico de hernia hiatal tipo III con manifestación clínica de neumonías a repetición, a la cual se le realizó una reparación del defecto herniario por vía laparoscópica junto con una funduplicatura tipo Nissen a $360^{\circ}$, consiguiendo la curación definitiva tempranamente. La poca frecuencia de esta variedad de hernia hiatal, y el abordaje mínimo invasivo con recuperación inmediata de la paciente hacen de este caso interesante para la literatura y comunidad médica. MÉD.UIS. 2017;30(2):83-8.

Palabras clave: Hernia Hiatal. Reflujo Gastroesofágico. Herniorrafia. Laparoscopia.

\section{Hiatal hernia: a diagnostic and therapeutic challenge. Case report}

\section{ABSTRACT}

The clinical presentation of a hiatal hernia, is a true diagnostic and therapeutic challenge for the health staff, because the presence of gastroesophageal reflux disease is not a synonym for hiatal hernia and on the other hand, a hiatal hernia not always debuts with gastroesophageal reflux disease, but rather manifests with extra abdominal symptoms such as chest pain or pulmonary manifestations. We present the case of a 66-year-old patient with a hiatal hernia type III with clinical manifestation of recurrent pneumonias, to whom the repair of the hernia defect was performed laparoscopically in conjunction with a 360 Nissen fundoplication, achieving total recovery quickly. The infrequency of this variety of hiatal hernia, and minimal invasive approach with immediate recovery of the patient make this case interesting for the medical literature and community. MÉD.UIS. 2017;30(2):83-8.

Keywords: Hernia, Hiatal. Gastroesophageal Reflux. Herniorrhaphy. Laparoscopy.

¿Cómo citar este artículo?: Pérez JJ, Viscarra JF, Ríos CG. Hernia hiatal: un desafío diagnóstico y terapéutico. A propósito de un caso. MÉD.UIS. 2017;30(2):83-8. 


\section{INTRODUCCIÓN}

La Hernia Hiatal $(\mathrm{HH})$ es una condición anatómica adquirida que se define como la protrusión o prolapso del estómago proximal o cualquier estructura no esofágica a través del hiato esofágico'. Las primeras descripciones de hernias diafragmáticas se realizaron en el año de 1580 por Ambrosio Paré, cirujano-barbero del Renacimiento que ocupaba el cargo de primer cirujano del rey Carlos IX de Francia².

Con el advenimiento de la radiología clínica se hizo evidente que la hernia diafragmática era una anomalía relativamente frecuente, y no siempre se relaciona con síntomas. La incidencia real de la $\mathrm{HH}$ en la población general es difícil de determinar debido a esta ausencia de síntomas ${ }^{3}$.

Según los criterios de Allison se han identificado los siguientes tipos de hernias hiatales:

- Tipo I: también llamadas hernias deslizantes, aparecen cuando el ligamento freno-esofágico (membrana freno-esofágica) no mantiene la Unión Gastro-Esofágica (UGE) en la cavidad abdominal. Así pues, el cardias se desplaza entre el mediastino posterior y la cavidad peritoneal.

- Tipo II: llamadas hernias paraesofágicas o rodantes, se producen cuando la UGE está anclada al abdomen pero el hiato, que suele ser grande, deja espacio para que las vísceras se desplacen al mediastino. La presión relativamente negativa del tórax facilita el desplazamiento visceral. Habitualmente, el fondo del estómago se desplaza al mediastino.

- Tipo III: también llamadas mixtas, son una combinación de la tipo I y II, en las que la UGE y el fondo se desplazan libremente por el mediastino.

- Tipo IV: aparecen cuando otros órganos en lugar o además del estómago se hernian a través del hiato. De forma característica estas hernias son grandes y contienen parte del colon o del bazo, además del estómago, dentro del tórax ${ }^{4}$.

La edad de presentación más frecuente es entre la $4^{a}$ y $6^{a}$ década de la vida y no existe diferencia en cuanto al sexo. Cerca del $85 \%$ de las $\mathrm{HH}$ verdaderas corresponden a las hernias de tipo I, las hernias de tipo II a un $14 \%$, las tipo III ocurren menos frecuentemente en un $0,8 \%$ y las hernia tipo IV en menos del $1 \% 3,10,11$.
Muchos pacientes con $\mathrm{HH}$ son asintomáticos $\mathrm{O}$ padecen síntomas muy leves, los cuales pueden estar relacionados con el reflujo gastroesofágico como regurgitación y pirosis en mayor frecuencia o disfagia, odinofagia, hematemesis y melena. Otras manifestaciones no relacionadas con reflujo son molestias bucofaríngeas, asma y dolor retroesternal.

Los síntomas atípicos producidos por reflujo gastroesofágico se presentan en un tercio de los pacientes y remedan una enfermedad respiratoria (disnea, tos, expectoración, ronquera), cardíaca (precordalgia), pancreática (dolor hemi-cinturón), gástrica (disfagia) o duodenal (dispepsia). Adicionalmente, en pacientes con el estómago intratorácico puede llegar a producirse obstrucción esofágica intermitente debido a la rotación que se ha originado a medida que el órgano migra hacia el tórax y se asocian manifestaciones respiratorias, tales como disnea por la compresión mecánica y neumonías recidivantes a causa de la aspiración de contenido gástrico ${ }^{5}$.

La baja frecuencia de presentación de la HH tipo III convierte en un verdadero desafío su diagnóstico, por lo que suele manifestarse como una masa mediastínica incidental en la radiografía de tórax, donde se ve proyectada por detrás del corazón, en el mediastino posterior, muchas veces con un nivel hidroaéreo. Con el uso de tomografía computada multidetector, en particular con reconstrucciones multiplanares, es posible caracterizar con detalle la anatomía de la hernia y definir de qué tipo se trata, establecer si existe rotación gástrica y de qué tipo es, evaluar la herniación de otras estructuras abdominales además del estómago y, por último, establecer la presencia de complicaciones como vólvulo o perforación gástrica.

El estudio baritado esofagogastroduodenal es fundamental para caracterizar la hernia en los casos en que se planea reparación quirúrgica, y es el mejor estudio para determinar el tamaño de la hernia y la posición de la UGE. La endoscopía digestiva alta juega un rol menor en el diagnóstico de hernia hiatal. Su mayor utilidad radica en confirmar obstrucción gástrica y descartar lesiones mucosas concomitantes que puedan sugerir isquemia ${ }^{6}$.

El tratamiento médico incluye una amplia gama de medicamentos como los antagonistas de los receptores $\mathrm{H}_{2}$ o los inhibidores de la bomba de 
protones que alivian en mayor o menor grado los síntomas. La mayoría de los autores acepta que el tratamiento definitivo de la $\mathrm{HH}$ es quirúrgico y se basa en dos elementos fundamentales: la reparación del hiato esofágico y la funduplicatura gástrica con el objetivo de crear un manguito de presión. Las indicaciones para la resolución quirúrgica son: hernia hiatal encarcelada, dolor torácico asociado a hernia hiatal mixta, paciente con severa deficiencia de hierro secundaria a erosiones o ulceraciones en la hernia hiatal, y hernia paraesofágica?. Dentro de las técnicas quirúrgicas la cirugía abierta presenta una alta tasa de complicaciones y precisa un tiempo de recuperación prolongado mientras que la cirugía laparoscópica ha demostrado ser factible y segura, presentando una menor estancia hospitalaria y menores tasas de complicaciones.

Se presenta el caso de una hernia hiatal tipo III, con herniación del estómago hacia el mediastino posterior, con el fin de discutir su manejo quirúrgico mínimo invasivo y su pronta recuperación para poder profundizar los conocimientos científicos de dicha patología debido a su baja frecuencia ${ }^{10,11}$.

\section{Presentación del Caso}

Paciente femenina, mestiza, de 66 años de edad, con antecedentes de hipertensión arterial en tratamiento, quien consultó por presentar cuadro clínico de neumonías a repetición, junto con dolor precordial, periodos esporádicos de epigastralgia y reflujo gastroesofágico. Dicha sintomatología se exacerbó en los últimos meses por lo que acudió a centro médico donde solicitaron radiografía estándar de tórax, en donde se evidenció presencia de nivel hidroaéreo en mediastino posterior superior al hemidiafragma izquierdo (Ver Figura 1).

Debido a esto la paciente fue derivada al Servicio de Cirugía Bariátrica del Omni Hospital, con el diagnóstico presuntivo de HH. Sele solicitó un estudio radiográfico contrastado de esófago, estómago y duodeno, donde se observó protrusión del estómago hacia el tórax a través del hiato esofágico con deslizamiento de la unión esofagogástrica en sentido cefálico y enrollamiento del estómago sobre la curvatura mayor (Ver Figura 2), reportándose $\mathrm{HH}$ mixta.

Con este diagnóstico se decidió realizar una reparación por vía laparoscópica. Dentro de los hallazgos quirúrgicos se encontró una $\mathrm{HH}$ mixta de $8 \mathrm{~cm}$ de diámetro cuyo contenido fue el fondo gástrico junto con epiplón mayor, razón por la cual se decidió realizar una corrección del defecto diafragmático junto con una funduplicatura tipo Nissen. (Ver Figura 3).

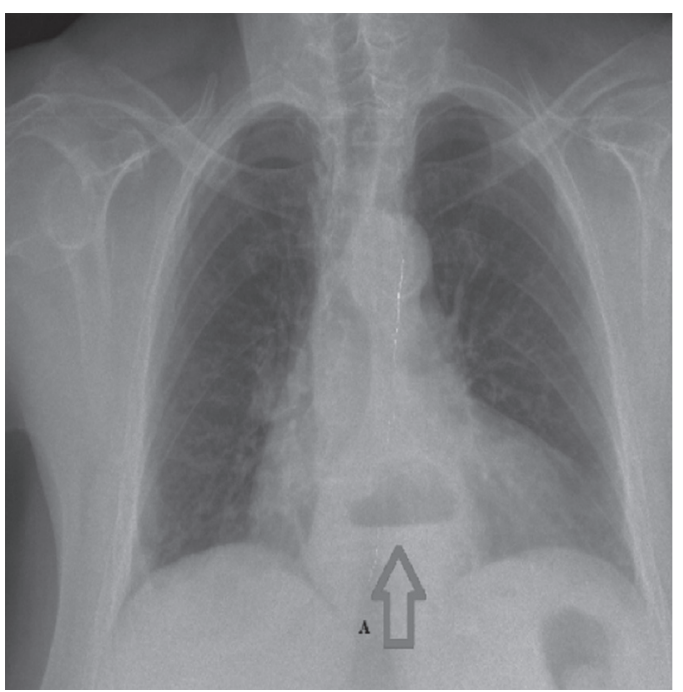

Figura 1: Radiografía estándar de tórax. A: Nivel hidroaéreo mediastino posterior

Fuente: autores

\section{PASOS DE LA TÉCNICA QUIRÚRGICA}

- Asepsia, antisepsia y colocación de campos quirúrgicos

- Diéresis: T1: 10mm para-umbilical izquierda con técnica abierta, T2: 10mm sub-xifoideo, T3: 10mm sub-costal izquierdo, T4: $5 \mathrm{~mm}$ flanco derecho, T5: $5 \mathrm{~mm}$ flanco izquierdo todos ellos bajo visión directa (Ver Figura 4).

- Liberar adherencias a nivel del ligamento falciforme y reducir la hernia diafragmática por vía laparoscópica, identificando el saco herniario.

- Identificar el pilar izquierdo del hiato y liberar adherencias del saco herniario hasta pilar derecho, realizar un túnel retro-esofágico y traccionar el mismo permitiendo identificar la curvatura mayor a nivel de borde inferior del bazo.

- Liberar los vasos cortos gástricos.

- Cerrar los pilares del hiato con 4 puntos separados de prolene 2 -o (Ver Figura 5A).

- Realizar maniobra retro-esofágica para traccionar el fondo gástrico y armar la válvula anti-reflujo con técnica de Nissen $360^{\circ}$ con prolene 2-0 (Ver Figura 5B).

Posterior al procedimiento quirúrgico se realizó estudio radiológico con contraste de control donde 
se evidenció corrección del defecto herniario, con el techo gástrico por debajo del hemi-diafragma izquierdo sin fuga del medio de contraste hacia la cavidad abdominal (Ver Figura 6).

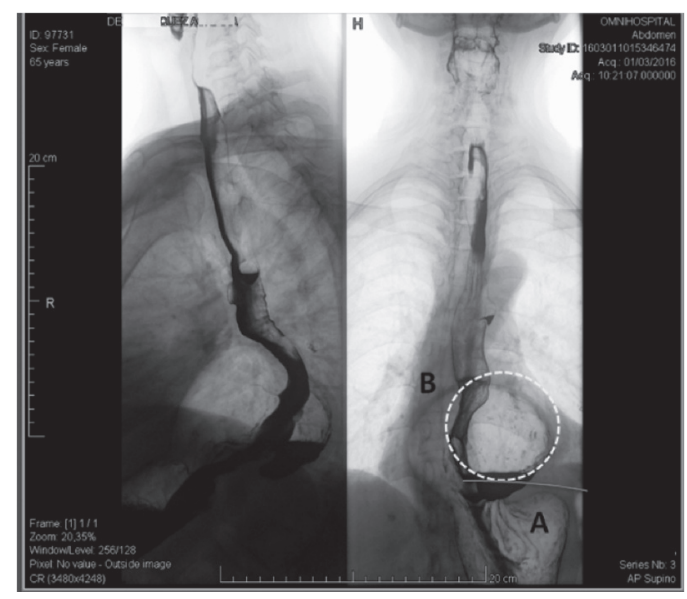

Figura 2: Estudio de tránsito esófagogastroduodenal con medio de contraste. A: Hemidiafragma izquierdo. B: Estómago en mediastino posterior

Fuente: autores

La paciente fue dada de alta médica en un periodo relativamente corto a las 48 horas posterior a la cirugía. Actualmente, después de seis meses de la intervención, la paciente se encuentró totalmente recuperada, sin sintomatología gastro-intestinal ni respiratoria.

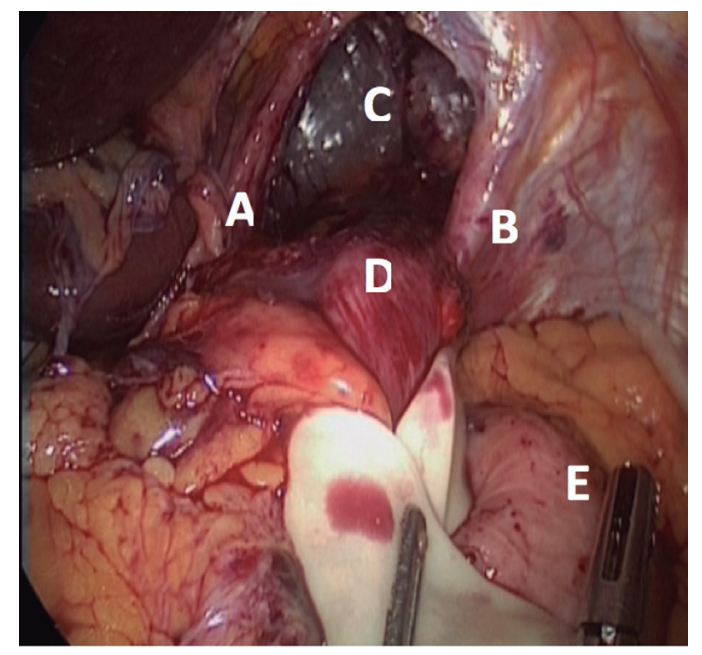

Figura 3: Hernia diafragmática. A: Pilar derecho. B: Pilar izquierdo. C: defecto herniario. D: Esófago. E: Fondo Gástrico Fuente: autores

\section{Discusión}

La HH tipo III presente en el caso es una patología poco frecuente con una incidencia inferior al $1 \%$, como ya se había mencionado. Ante la sospecha diagnóstica de una $\mathrm{HH}$ o el hallazgo incidental de la misma en una radiografía de tórax, la elección del examen complementario para el diagnóstico definitivo es muy importante ya que la solicitud de exámenes de gabinete extensos puede provocar retardo en el diagnóstico y derroche de recursos económicos. Por tal motivo hay que recordar que el gold standard en el diagnóstico de $\mathrm{HH}$ es el estudio radiográfico contrastado de esófago, estómago y duodeno, dicho estudio se puede complementar con una endoscopía digestiva alta y una biopsia gástrica para descartar patologías concomitantes ${ }^{8}$.

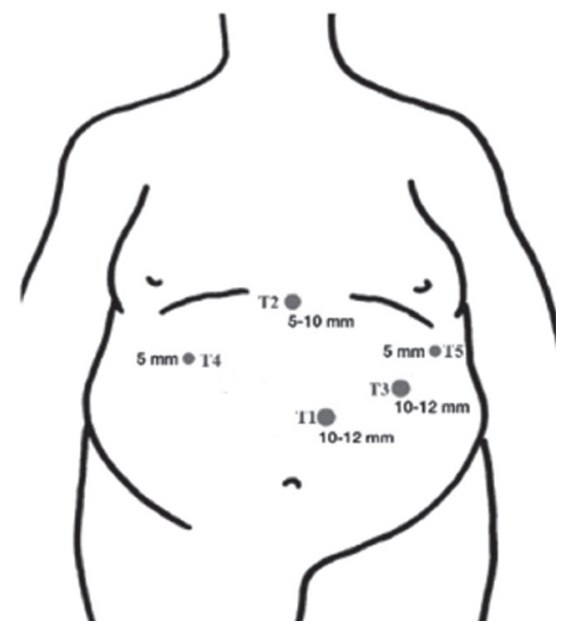

Figura 4: Esquema de sitio de inserción de los trocares a nivel abdominal

Fuente: autores
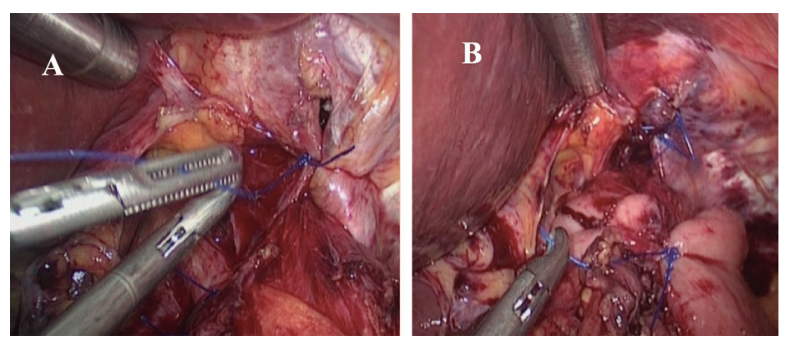

Figura 5: Técnica quirúrgica. A: Fijación pilares diafragmáticos. B: Funduplicatura Nissen $360^{\circ}$

Fuente: autores

El tratamiento médico es poco eficiente en este tipo de patología ya que conlleva un amplio cambio orgánico y funcional. El abordaje quirúrgico se convierte en el tratamiento definitivo y este puede ser transabdominal o transtorácico ya sea en forma abierta o laparoscópica con un alivio de los síntomas en un $80 \%$ al $100 \%$ de los pacientes en un periodo de seguimiento de diez años?

Con respecto al tratamiento quirúrgico se puede optar por la cirugía abierta que conlleva un riesgo de 
morbimortalidad y estancia hospitalarias mucho más elevados de hasta nueve días más que el tratamiento realizado con la técnica de video laparoscopía. El advenimiento y desarrollo de los procedimientos quirúrgicos antirreflujo por vía laparoscópica han demostrado su superioridad por presentar una mortalidad reducida $0-0,5 \%$, baja morbilidad (infección sitio quirúrgico, dolor post-quirúrgico) 2-13\% y una estancia hospitalaria reducida de hasta tres días y con igual seguridad-efectividad que los procedimientos por técnica abierta, ofreciendo una mejor opción quirúrgica para aquellos pacientes con enfermedad severa o asociada a complicaciones9.

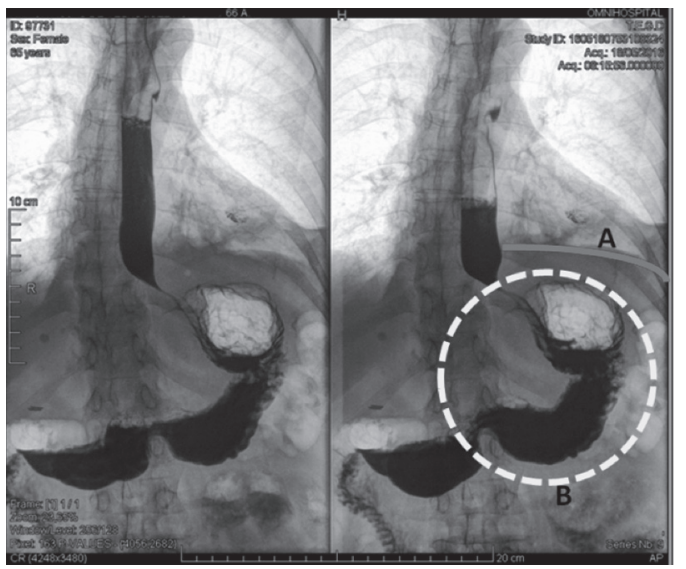

Figura 6: Estudio de tránsito esófago-gastroduodenal con medio de contraste. A: Hemidiafragma izquierdo. B: Estómago Fuente: autores

En la actualidad se acepta que el procedimiento quirúrgico debe incluir la reducción de la hernia, excisión del saco herniario, corrección del defecto diafragmático, y la mayoría de los autores defienden la realización de un procedimiento antirreflujo (Nissen, Hill, Toupet), basados en que dos tercios de los pacientes con hernia diafragmática tienen evidencia objetiva de reflujo gastroesofágico, además al movilizar el hiato probablemente se alteran los mecanismos intrínsecos antirreflujo.

Según el trabajo de Granados RJJ y colaboradores la funduplicatura tipo Nissen es la técnica más efectiva. En este se evaluaron técnicas de funduplicatura del esófago distal, tomando como principal la técnica de Nissen. Se obtuvieron como resultado que la mejoría clínica con la técnica de Nissen fue del $80 \%$, Toupet $70 \%$ y Door $50 \%$. Estos datos indican que la técnica tipo Nissen es de utilidad para la mejora de las sintomatologías atípicas del reflujo gastroesofágico. A pesar de lo expuesto la técnica quirúrgica de Nissen tiene ciertas complicaciones como disfagia, incapacidad para eructar y vomitar, llenura postprandial, dolor y flatulencia que deben tomarse en cuenta, ya que aparecen en un 19\% de los pacientes. En el trabajo de Aurora Carrasquer se notó una mejoría clínica en la sintomatología típica y atípica en el $86 \%$ de los pacientes sometidos a la operación, de los cuales el $76 \%$ tuvo una buena calificación en los test de calidad de vida, lo cual refleja la utilidad de la técnica de Nissen. Demostrando que la funduplicatura por la técnica de Nissen tiene más efectividad para el control del reflujo que las funduplicaturas parciales ${ }^{12}$.

Por lo expuesto anteriormente, esta fue la conducta terapéutica que se adoptó con la paciente, obteniendo su egreso hospitalario temprano a las 48 horas y a pesar de las complicaciones descritas en la literatura con la funduplicatura de Nissen, durante el seguimiento del caso que hasta el momento es de seis meses no se han reportado complicaciones relacionadas con la técnica quirúrgica, pero si una mejoría notable en su calidad de vida.

\section{CONCLUSIONES}

La HH mixta es una patología poco frecuente cuya manifestación clínica por lo general suele ser extra abdominal y cuyo hallazgo imagenológico es incidental. El tratamiento quirúrgico de elección es por vía laparoscópica ya que reduce el riesgo de infección del sitio quirúrgico, mejor tolerancia al dolor post operatorio, menor estancia hospitalaria y una reinserción laboral más temprana. La técnica quirúrgica de elección es la funduplicatura tipo Nissen $360^{\circ}$ que produce una respuesta de satisfacción en los pacientes de hasta el 95\%, sin embargo la técnica utilizada dependerá de la experiencia del cirujano y de los hallazgos quirúrgicos al momento de la cirugía.

\section{Referencias BibLIográficas}

1. Pereira JG, Roque R, Martínez MA, Torres R, Barreras J, López AB. Cirugía videolaparoscópica en la hernia hiatal gigante. Rev Cubana Cir. 2015;54(2):148-156.

2. Depalma D. Hernia hiatal. Rev Hosp Niños BAires. 2012;54(246):184-188

3. Schwartz Brunicard C. Principios de cirugía. México, D.F.: McGraw-Hill Interamericana; 2008. p.842-5.

4. Sabiston.Townsend C. Tratado de cirugía. Amsterdam, Madrid: Elsevier; 2013. 1067-70.

5. Casas García J. Enfermedades quirúrgicas del abdomen: Hernia hiatal [Internet]. En: García Gutierrez A. Cirugía. Tomo III. Cuba: 2007 [Citado 22 May 2016]. Disponible en: http://gsdl. bvs.sld.cu/cgi-bin/library?e=d-00000-00---off-0cirugia--00-0---0-10-0---0---0direct-10---4-------0-11--11-zh-50---20-about---000-1-00-0-0-11-1-0gbk-00\&cl=CL3.2\&d=HASH018a7195c694eb 5 fe10cd174.4.4\&hl $=0 \& \mathrm{gc}=0 \& \mathrm{gt}=0$

6. De Grazia K J, Godoy Z M, Cavallo B Í, Cortés A C. Hernia hiatal gigante con estómago intratorácico: Reporte de un caso 
y revisión de la literatura. Rev. chil. radiol. 2012;18(4):179-83.

7. [Internet]. 2016 [Citado 01 Sep 2016]. Disponible en: http:// med.javeriana.edu.co/publi/vniversitas/serial/v41n4/0043\%20 hernia.pdf

8. Madriz Meza W, Alvarado Rodríguez VH, Hernández JJ. Diagnóstico radiológico de hernia hiatal (Revisión bibliográfica y presentación de casos). Revista Médica de Costa Rica y Centroamerica [Internet]. 2008 [citado 01 Sep 2016];65(583):103-8. Disponible en: http://www.binasss.sa.cr/ revistas/rmcc/583/art5.pdf

9. Bejarano Cuéllar W. Hernia Hiatal. Universitas Médicas [Internet]. 2000 [Citado 27 Sep 2016];41(4): Disponible en: http://webcache.googleusercontent.com/ search?q=cache:mitNXX7YiAcJ:med.javeriana.edu. co/publi/vniversitas/serial/v41n4/0043\%2520hernia $\mathrm{pdf}+\& \mathrm{~cd}=1 \& \mathrm{hl}=\mathrm{es} \& \mathrm{ct}=\mathrm{clnk} \& \mathrm{gl}=\mathrm{ec}$

10. Unidad-gastroenterologia. Hernia Hiatal [Internet]. 2016 [Citado 02 Dic 2016]. Disponible en: http://unidad-gastroenterologia. com/hernia-hiatal.html

11. Braghetto IM, Korn OB, Burdiles P, Debandi A, Valladares H, Brunett L. Hernias hiatales verdaderas: tratamiento quirúrgico por vía laparoscópica. Rev. Chilena de Cirugía. 2002;54(6):62838.

12. Granados Romero JJ, Cortés González LD, Zacarías Ezzat JR, Tapia Jurado J, Athié Gutierrez C, Aguirre Cazares ML. Resultados de diversas técnicas de funduplicaturas en la resolución de sintomatología respiratoria atípica. Revista de la Asociación Mexicana de Cirugía Endoscópica. 2013;14(1):6-9. 